\title{
SLC26A4 mutation spectrum associated with DFNB4 deafness and Pendred's syndrome in Pakistanis
}

\author{
Saima Anwar ${ }^{1}$, Saima Riazuddin ${ }^{2}$, Zubair M Ahmed ${ }^{2}$, Saba Tasneem ${ }^{1}$, Ateeq-ul-Jaleel ${ }^{1}$, Shahid Y Khan ${ }^{1}$, \\ Andrew J Griffith ${ }^{3}$, Thomas B Friedman ${ }^{2}$ and Sheikh Riazuddin ${ }^{1}$
}

Pendred's syndrome (PDS) is an autosomal-recessive disorder characterized by sensorineural hearing loss and goiter. PDS is caused by mutations of the $S L C 26 A 4$ gene encoding pendrin, a transmembrane exchanger of $\mathrm{Cl}^{-}, \mathrm{I}^{-}$and $\mathrm{HCO}_{3}^{-}$, which is expressed in the thyroid and inner ear. SLC26A4 mutations can also be associated with non-syndromic deafness, DFNB4. The goal of our study was to define the identities and frequencies of SLC26A4 mutations in 563 large, consanguineous Pakistani families segregating severe-to-profound recessive deafness. Sequence analyses of SLC26A4 in 46 unreported families segregating deafness linked to DFNB4/PDS revealed 16 probable pathogenic variants, 8 of which are novel. The novel variants include three missense substitutions (p.R24L, p.G139V and p.V231M), two splice site mutations (c.304+2T $>C$ and c.1341+3A $>$ C), one frameshift (p.C565MfsX8) and two different genomic deletions affecting exons 1-2 and 11-18. Each of six pathogenic variants (p.V239D, p.Q446R, p.S90L, p.Y556C, p.R24L and p.K715N) was found in more than one family and haplotype analyses suggest that they are founder mutations. Combined with earlier reported data, SLC26A4 mutations were identified in 56 (7.2\%; 95\% Cl: 5.6-9.2\%) of 775 families. Therefore, SLC26A4 mutations are the most common known cause of genetic deafness in this population. As p.V239D (30\%), p.S90L (18\%) and p.Q446R (18\%) account for approximately two-third of the mutant alleles of SLC26A4, hierarchical strategies for mutation detection would be feasible and cost-efficient genetic tests for DFNB4 deafness and PDS in Pakistanis.

Journal of Human Genetics (2009) 54, 266-270; doi:10.1038/jhg.2009.21; published online 13 March 2009

Keywords: deafness; DFNB4; Pakistan; Pendred's syndrome; SLC26A4

\section{INTRODUCTION}

Pendred's syndrome (PDS) (OMIM\# 274600) is classically described as bilateral sensorineural hearing loss and thyroid enlargement (goiter). Structural malformations of the inner ear, including enlarged vestibular aqueducts and Mondini defects of the cochlea, are highly penetrant features of PDS. ${ }^{1}$ PDS is caused by mutations of the SLC26A4 gene, which encodes pendrin. ${ }^{2}$ Pendrin is a transmembrane exchanger of $\mathrm{Cl}^{-}, \mathrm{I}^{-}$and $\mathrm{HCO}_{3}^{-}$, which is expressed in the thyroid and inner ear. ${ }^{3}$ SLC26A4 mutations have also been implicated in nonsyndromic deafness, DFNB4 (OMIM\# 600791), ${ }^{4}$ although the genotypic and phenotypic overlap of PDS and DFNB4 has led to uncertainty in their nosology. ${ }^{5,6}$ In 1965 , Fraser $^{7}$ phenotypically estimated that PDS accounts for $\sim 10 \%$ of hereditary hearing loss. A more recent genotypic survey of different East and South Asian deaf populations showed that SLC26A4 mutations account for at least $5 \%$ of cases of severe-to-profound deafness. ${ }^{8}$ These and other studies collectively suggest that SLC26A4 mutations are one of the most common causes of genetic deafness in many populations, including Pakistanis. ${ }^{8}$
To date, more than 150 SLC26A4 mutations have been identified in patients with PDS, DFNB4 or enlarged vestibular aqueduct. ${ }^{9}$ The most prevalent mutations tend to be specific to one or a few related ethnic groups. ${ }^{8,10,11}$ p.T416P and IVS8+1G $>A$ are common in Northern Europe, whereas p.H723R and IVS7-2A $>\mathrm{G}$ are the most common in East Asia. ${ }^{8,11,12}$ We earlier identified mutant alleles of SLC26A4 in 10 (4.7\%; 95\% CI: $2.6-8.4 \%)$ of 212 Pakistani families segregating recessive deafness, which were ascertained through the National Centre of Excellence in Molecular Biology (NCEMB) in Pakistan. ${ }^{8}$ p.S90L was the most commonly detected mutation in that initial study. The different spectra of SLC26A4 mutations in different ethnic populations should be amenable to ethnically based, hierarchical mutation detection strategies that are rapid and cost-efficient. ${ }^{13}$

The NCEMB study population is a robust model for genotypic studies of non-syndromic and syndromic deafness phenotypes in Pakistan. ${ }^{8,14-19}$ The goal of our study was to define the identities, frequencies and origins of SLC26A4 pathogenic variants in a much larger cohort of 775 Pakistani families segregating recessive severe-toprofound deafness with or without goiter. We show that SLC26A4

${ }^{1}$ National Center of Excellence in Molecular Biology, Punjab University, Lahore, Pakistan; ${ }^{2}$ Laboratory of Molecular Genetics, Section on Human Genetics, National Institute on Deafness and Other Communication Disorders, National Institutes of Health, Rockville, MD, USA and ${ }^{3}$ Otolaryngology Branch, National Institute on Deafness and Other Communication Disorders, National Institutes of Health, Rockville, MD, USA

Correspondence: Dr S Riazuddin, National Centre of Excellence in Molecular Biology, University of the Punjab, 87-West Canal Bank Road, Thokar Niaz Baig, Lahore 53700, Pakistan.

E-mail: riaz@|hr.comsats.net.pk

Received 31 October 2008; revised 27 January 2009; accepted 9 February 2009; published online 13 March 2009 
mutations are a common cause of genetic deafness among Pakistanis and their distinctive spectrum lends itself to hierarchical detection strategies.

\section{MATERIALS AND METHODS}

Subjects and phenotype analysis

This study was approved by the IRB (International Review Board) at the National Centre of Excellence in Molecular Biology (NCEMB), Lahore, Pakistan (FWA00001758), and the Combined Neuroscience IRB at the National Institutes of Health, USA (OH-93-N-016). Written informed consent was obtained from adult participants and from parents of minor participants of 775 Pakistani families. Hearing was evaluated using pure-tone audiometry at octave frequencies with intensities of up to $100 \mathrm{dBHL}$. Vestibular function was evaluated by tandem gait and Romberg testing. All family members were examined by a local physician to detect palpable goiter (Supplementary Table 1).

\section{Genotype analysis}

Genomic DNA was extracted from $10 \mathrm{ml}$ of peripheral venous blood as described. ${ }^{20}$ Primers for PCR (polymerase chain reaction) amplification, SLC26A4 sequencing, single nucleotide polymorphism genotyping and ARMS (amplification refractory mutation system) were designed using Primer3 (http://frodo.wi.mit.edu/cgi-bin/primer3/primer3_www.cgi/). Three fluorescent-labeled microsatellite markers (D7S2420, D7S2459 and D7S2456) linked to SLC26A4 were PCR-amplified and analyzed as described. ${ }^{20}$ We designed ARMS primers with mismatches to differentially amplify the p.V239D or wildtype alleles. The four primers were PDS6F (wild-type-specific) 5'-GTGCTGGT

Table 1 Mutation spectrum of SLC26A4 in Pakistani NCEMB families

\begin{tabular}{|c|c|c|c|}
\hline Nucleotide variation & Effect & Exon & $\begin{array}{l}\text { Number } \\
\text { of families }\end{array}$ \\
\hline c.-23177_c.164+1027del24368ins7 & Genomic deletion & $1-2$ & 1 \\
\hline c. $71 \mathrm{G}>\mathrm{T}$ & p.R24L & 2 & 2 \\
\hline c. $170 \mathrm{C}>\mathrm{A}$ & p.S57X & 3 & 1 \\
\hline c. $269 \mathrm{C}>\mathrm{T}$ & p.S90L & 3 & 5 \\
\hline c. $304+2 \mathrm{~T}>\mathrm{C}$ & p.M103KfsX4a & 3 & 1 \\
\hline c. $416 \mathrm{G}>\mathrm{T}$ & p.G139V & 5 & 1 \\
\hline c. $694 \mathrm{G}>\mathrm{A}$ & p.V231M & 6 & 1 \\
\hline c.716 T>A & p.V239D & 6 & 16 \\
\hline c. $1115 \mathrm{C}>\mathrm{T}$ & p.A372V & 9 & 1 \\
\hline c. $1337 A>G$ & p.Q446R & 11 & 10 \\
\hline c. $1341+3 A>C$ & p.G439VfsX19a & 11 & 1 \\
\hline c. $1363 \mathrm{~A}>\mathrm{T}$ & p.I455F & 12 & 1 \\
\hline c. $1667 A>G$ & p.Y556C & 15 & 2 \\
\hline c.1692_1693insA & p.C565MfsX8 & 15 & 1 \\
\hline c.1264-477_2090-4927del11202 & Genomic deletion & $11-18$ & 1 \\
\hline c. $2145 \mathrm{G}>\mathrm{T}$ & p.K715N & 19 & 2 \\
\hline
\end{tabular}

Abbreviation: NCEMB, National Centre of Excellence in Molecular Biology. aEffects on protein (GenBank Accession \# AAC51873) are predicted by GenScan.
CTCACAGCTAAAGATAGA-3', PDS6R (p.V239D-specific) 5-GTAGTTTTTG GTTGAAACATTGACGA-3'，PDS6F (outer) 5'-TTTCCAGAGAGTAGGTTTC TATCTCAGG- $3^{\prime}$ and PDS6R (outer) 5-CCAGACTCAGAGAATGAATACAATT GTT-3'. Amplifications comprised 35 step-cycles with an annealing temperature of $58^{\circ} \mathrm{C}, 50 \mathrm{ng}$ genomic DNA and standard amplification conditions, except that we used a higher concentration $(16 \mu \mathrm{M})$ of outer primers compared with that of mutant- and wild-type-specific inner primers $(8 \mu \mathrm{M})$. PCR products were separated by $2 \%$ agarose gel electrophoresis and visualized with ethidium bromide.

\section{Statistical analysis}

Differences in single nucleotide polymorphism genotype distributions between p.V239D homozygotes and ethnically matched, normal-hearing controls were analyzed using Fisher's exact test.

\section{RESULTS}

The study participants comprised 563 families segregating severe-toprofound, prelingual- or congenital-onset, recessive deafness ascertained through the NCEMB after the original 212 families reported by Park et al. ${ }^{8}$ We identified 46 additional families co-segregating deafness (Supplementary Table 1) with short tandem repeat markers linked to SLC26A4. Sequence analysis of SLC26A4 revealed 16 probable pathogenic variants co-segregating with deafness in each of the 46 families (Table 1).

Novel variants include missense substitutions, p.R24L (c.71G > T), p.G139V (c.416G > T) and p.V231M (c.694G > A). All of these affect amino acid residues that are conserved among pendrin orthologs (Figure 1). We identified two novel splice site variants, c.304+2T $>\mathrm{C}$ and $c .1341+3 \mathrm{~A}>\mathrm{C}$ (Table 1), which are predicted to disrupt conserved splice donor site consensus sequences. ${ }^{21}$ We found one frameshift (c.1692_1693insA) predicted to prematurely truncate the protein (p.C565MfsX8). There were two novel genomic deletions of SLC26A4: c.-23177_164+1027del24368ins7 deletes exons 1 and 2 and c.1264477_2090-4927del11202 encompasses exons 11-18. Alu repeats at the breakpoints of c.23177_164+1027del24368ins7 may have led to its origin through unequal crossing over. ${ }^{22-25}$ These novel mutations were not detected in 192 chromosomes from geographically and ethnically matched, normal-hearing participants.

We also identified eight earlier reported pathogenic variants: c.716T >A (p.V239D), c.269C > T (p.S90L), c.1363A > T (p.I455F), c.1667A $>$ G (p.Y556C), c.1337A $>$ G (p. Q446R), c.1115C $>$ T (p.A372V), c.170C >A (p.S57X) and c.2145G $>$ T (p.K715N). ${ }^{8,11,26,27}$ p.V239D, p.S90L, p.Y556C, p. Q446R and p.K715N were detected in more than one family (Tables 1 and 2). p.A372V was detected in compound heterozygosity with c.304+2T $>$ C.

We performed haplotype analyses of linked short tandem repeat markers and single nucleotide polymorphisms (Table 1 and Figure 2) to identify potential founder effects for recurrent variants of SLC26A4. The results are consistent with common ancestors for each of the six alleles (Table 2). Although 16 independent p.V239D chromosomes did

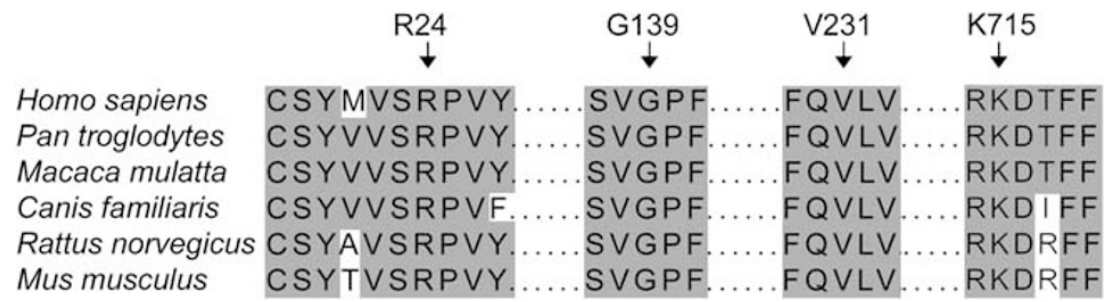

Figure 1 Multiple sequence alignment of pendrin from human, chimpanzee, monkey, dog, rat and mouse. Amino acid residues R24, G139, V231 and K715 of human pendrin are conserved among orthologs. Non-contiguous portions of pendrin are separated by dotted lines. 
not share a common D7S2420/D7S2456 haplotype, the intragenic SLC26A4 marker (D7S2459) allele is the same on all 16 chromosomes. Highly significant associations between p.V239D and each of seven closely linked single nucleotide polymorphisms (Figure 2, Table 3) support the hypothesis that p.V239D is a founder mutation that arose in a common ancestor.

We compared the frequencies of all known mutant alleles of SLC26A4 (Figure 3) among the NCEMB families. Exons 3, 6 and 11 collectively account for the majority $(72 \% ; 41 / 57)$ of mutant alleles (Figure 3). As p.V239D accounts for 30\% (17/57; 95\% CI: $19.5-$ $42.7 \%$ ) of the mutant alleles, we developed a tetra-primer ARMS assay to rapidly and inexpensively detect p.V239D carriers and homozygotes (Figure 4). The sensitivity and specificity of this assay were confirmed on multiple DNA samples with genotypes established by direct sequencing (Figure 4).

\section{DISCUSSION}

Taken together with our earlier result, ${ }^{8}$ SLC26A4 mutations account for $7.2 \%$ (56 of 775 families; 95\% CI: 5.6-9.2\%, Table 4) of deafness in

Table 2 SLC26A4 mutation-linked STR haplotypes

\begin{tabular}{|c|c|c|c|c|}
\hline \multirow[b]{2}{*}{ Mutation } & \multicolumn{3}{|c|}{ STR allele size (bp) } & \multirow[b]{2}{*}{ PKDF family code } \\
\hline & D7S2420 & D7S2459 & D7S2456 & \\
\hline \multirow[t]{2}{*}{ p.Q446R } & 278 & 134 & 239 & 049,812 \\
\hline & 278 & 134 & 247 & $\begin{array}{l}293,354,396,398,451, \\
453,486,775\end{array}$ \\
\hline \multirow[t]{8}{*}{ p.V239D } & 276 & 140 & 239 & 146 \\
\hline & 278 & 140 & 243 & 235 \\
\hline & 280 & 140 & 247 & 705, 709 \\
\hline & 282 & 140 & 239 & 263 \\
\hline & 282 & 140 & 241 & 427 \\
\hline & 284 & 140 & 243 & $\begin{array}{l}042,209,485,541,700, \\
706,846\end{array}$ \\
\hline & 284 & 140 & 239 & 134,278 \\
\hline & 284 & 140 & 241 & 259 \\
\hline \multirow[t]{2}{*}{ p.S90L } & 276 & 140 & 239 & $347,726,734,831$ \\
\hline & 276 & 140 & 241 & 376 \\
\hline \multirow[t]{2}{*}{ p.K715N } & 276 & 138 & 241 & 143 \\
\hline & 276 & 138 & 239 & 502 \\
\hline p.R24L & 274 & 138 & 239 & 372,772 \\
\hline p.Y556C & 282 & 138 & 241 & 172,804 \\
\hline
\end{tabular}

Abbreviation: STR, short tandem repeat. the NCEMB Pakistani study population. These results indicate that SLC26A4 mutations are a significant cause of recessive hearing loss in the Pakistani population (Table 4). Our estimated SLC26A4 mutation prevalence is not significantly different from that of other studies in different populations. SLC26A4 mutations account for $8.95 \%$ of prelingual deafness in a study of 514 patients from Northern China. ${ }^{10}$ In a few eastern and central regions of China, the c.IVS7$2 \mathrm{~A}>\mathrm{G}$ allele alone accounts for $8-12 \%$ of individuals with hearing loss. ${ }^{12}$ In Koreans and Mongolians, SLC26A4 mutations account for 6.5\% (95\% CI: $2.7-14.2)$ and $2.1 \%$ (95\% CI: $0.7-5.5)$ of deafness, respectively. ${ }^{8}$ In Japan, mutations of GJB2, SLC26A4, CDH23 and the mitochondrial $12 \mathrm{~S}$ rRNA are major causes of hearing loss. ${ }^{28}$ We are yet to evaluate GJB2 in our NCEMB cohort of deaf participants from Pakistan. However, in another study, ${ }^{29}$ mutations of GJB2 also make a significant contribution to hearing loss in Pakistan (Table 4).

Probands of the NCEMB deafness cohort are usually students in schools for the hearing impaired who are often profoundly deaf from birth. This strategy for ascertaining families segregating profound hearing loss will miss families segregating mild or delayed-onset hearing loss. Thus, we have likely overlooked some of the phenotypic variability because of mutations of SLC26A4 in the Pakistani population and have underestimated the prevalence of DFNB4/PDS. We have also not evaluated participants for enlarged vestibular aqueduct. For economic and geographical reasons, it is not feasible to routinely do a computed tomography scan to detect enlarged vestibular aqueduct. However, PCR-based assays that are used to detect mutations of SLC26A4 are quick and affordable.

Table 3 SNP analysis of c.716T $>$ A (p.V239D) homozygotes and ethnically matched normal controls

\begin{tabular}{|c|c|c|c|c|c|c|c|c|}
\hline \multirow[b]{3}{*}{$d b S N P I D$} & \multirow[b]{3}{*}{$\begin{array}{c}\text { Position } \\
\text { (bp) }\end{array}$} & \multirow[b]{3}{*}{ Allele } & & \multicolumn{4}{|c|}{ Number of chromosomes } & \multirow[b]{3}{*}{$\mathrm{P}$-value ${ }^{\mathrm{a}}$} \\
\hline & & & & \multicolumn{2}{|c|}{$\begin{array}{c}\text { Case } \\
(n=16)\end{array}$} & \multicolumn{2}{|c|}{$\begin{array}{l}\text { Control } \\
(n=32)\end{array}$} & \\
\hline & & & e 1 Allele 2 & $1 / 1$ & $2 / 2$ & $1 / 1$ & $2 / 2$ & \\
\hline rs6949189 & 107095577 & C & G & 0 & 32 & 32 & 30 & $3.0 \times 10^{-8}$ \\
\hline rs2395910 & 107097048 & $\mathrm{G}$ & $A$ & 0 & 32 & 31 & 25 & $2.0 \times 10^{-8}$ \\
\hline rs11769313 & 107101183 & $\mathrm{G}$ & $A$ & 32 & 0 & 36 & 28 & $8.5 \times 10^{-7}$ \\
\hline rs13232692 & 107104946 & G & A & 32 & 0 & 26 & 36 & $2.0 \times 10^{-9}$ \\
\hline rs10240818 & 3107105651 & G & $\mathrm{T}$ & 0 & 32 & 41 & 23 & $1.0 \times 10^{-10}$ \\
\hline rs6953220 & 107110427 & $\mathrm{~T}$ & $G$ & 0 & 32 & 25 & 37 & $3.6 \times 10^{-6}$ \\
\hline rs6970857 & 107110445 & $\mathrm{C}$ & G & 0 & 32 & 31 & 29 & $3.8 \times 10^{-8}$ \\
\hline
\end{tabular}

Abbreviation: SNP, single nucleotide polymorphism. aFisher's exact test.

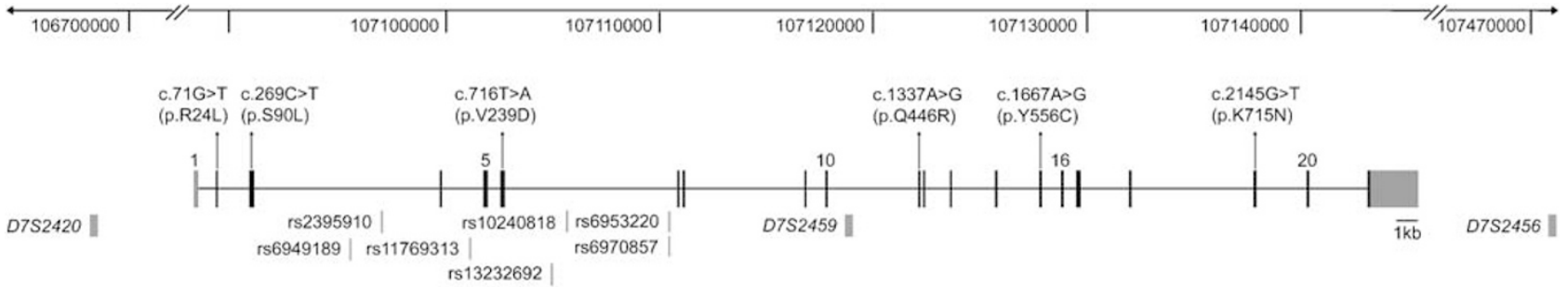

Figure 2 Physical map of human chromosome 7q22.3. The schematic diagram of SLC26A4 shows the location of six common mutations, seven single nucleotide polymorphisms (SNPs) and three (short tandem repeat) STR markers used for haplotype analyses. Positions are numbered according to the UCSC genome browser (March 2006 assembly, build 36.1, http://genome.cse.ucsc.edu/cgi-bin/hgGateway). 


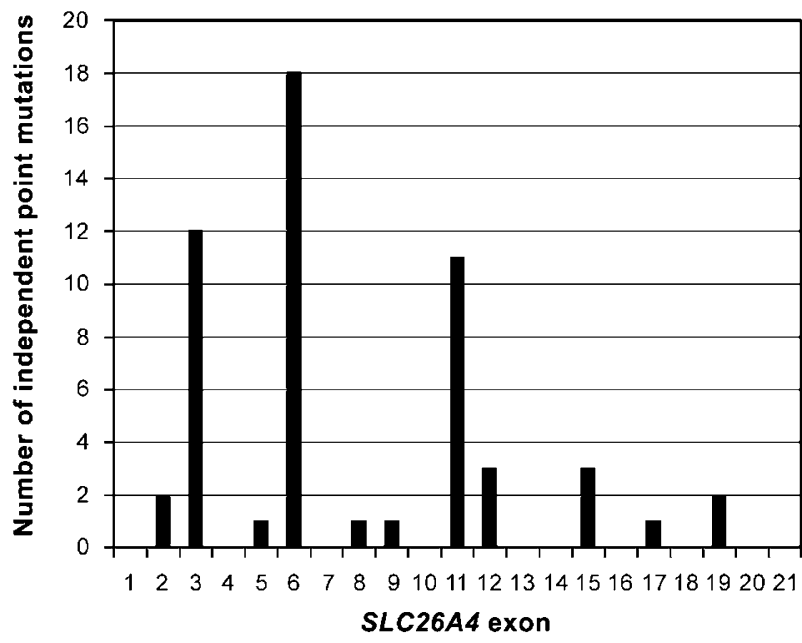

Figure 3 Exonic locations and frequencies of pathogenic SLC26A4 point mutations in the Pakistani National Centre of Excellence in Molecular Biology (NCEMB) study population, including families from Park et al. 2003. ${ }^{8}$ Exons 3, 6 and 11 contain the majority (72.0\%) of detected mutations. Besides the 55 point mutations, we detected two deletions (c.-23177_c.164+1027del24368ins7 and c.1264-477_2090-4927del11202) that encompass more than one exon (Table 1 ).

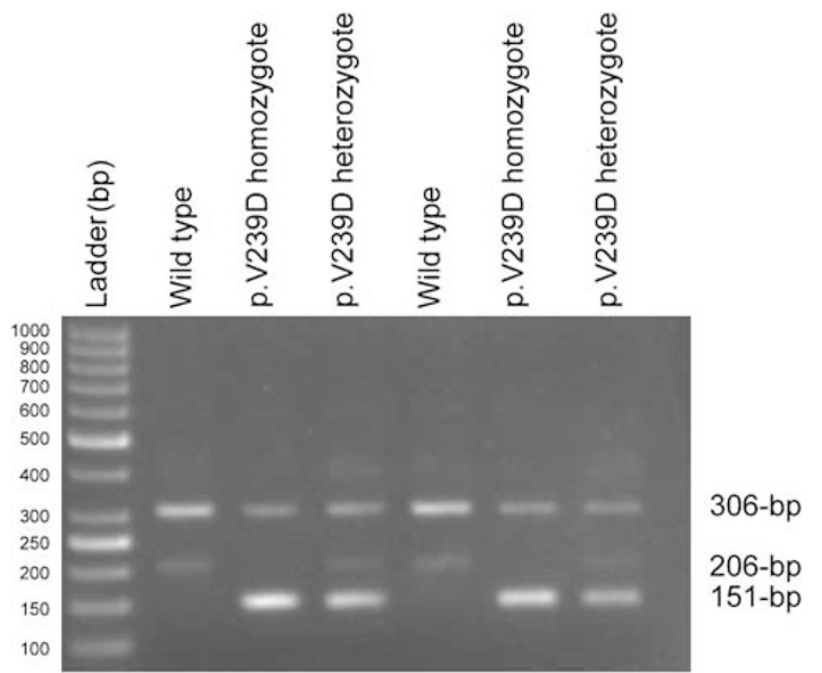

Figure 4 Results of Tetra Primer amplification refractory mutation system (ARMS) assay for detection of c. $\overline{7} 16 \mathrm{~T}>\mathrm{A}$ (p.V̄239D). Polymerase chain reaction (PCR) amplification using the outer primers generates a 306-bp positive control amplimer in all samples. Nested allele-specific primers generate a 206-bp product from the wild-type allele, whereas the p.V239D allele results in a 151-bp amplicon.

Ten of the mutations of SLC26A4 identified in this study were private (found in single families). However, six mutations account for more than half of the mutant alleles that we found in Pakistani families (Table 1). We observed a distinctive spectrum of SLC26A4 mutations that did not include alleles prevalent in other populations, such as c.IVS7-2A $>\mathrm{G}$ (Chinese and Koreans), p.H723R (Koreans and Japanese), p.V138F (Germans) or c.766-2A $>$ G (Taiwanese) ${ }^{8,30-32}$ As three alleles (p.S90L, p.V239D and p.Q446R) account for $\sim 66 \%$ of the cases of DFNB4/PDS deafness in the NCEMB study population, hierarchical mutation screening would be a cost-effective approach to molecular diagnosis. For example, testing for p.V239D would provide
Table 4 Contribution of different genes to hereditary hearing loss in Pakistani NCEMB cohort of families

\begin{tabular}{|c|c|c|c|c|}
\hline Gene & Locus & $\begin{array}{c}\text { Percentage } \\
\text { (fraction) of } \\
\text { Families }\end{array}$ & $95 \% \mathrm{Cl}$ & References \\
\hline SLC26A4 & $D F N B 4 / P D S$ & $7.2(56 / 775)$ & $5.6-9.2$ & This study; Park et al. ${ }^{8}$ \\
\hline GJB2 & $D F N B 1$ & $6.1(12 / 196)$ & $3.5-10.3$ & Santos et al. 29 \\
\hline$T M C 1$ & $D F N B 7 / 11$ & $3.4(19 / 557)$ & $2.2-5.3$ & Chishti et al. ${ }^{15}$ \\
\hline MY015A & DFNB3 & $3.3(20 / 600)$ & $2.2-5.1$ & Nal et al. ${ }^{33}$ \\
\hline OTOF & $D F N B 9$ & $2.3(13 / 557)$ & $1.4-4.0$ & Choi et al. ${ }^{34}$ \\
\hline TRIOBP & DFNB28 & $1.6(9 / 557)$ & $0.8-3.0$ & Riazuddin et al. ${ }^{18}$ \\
\hline \multirow[t]{2}{*}{ TRIC } & DFNB49 & $1.1(3 / 284)$ & $0.3-3.0$ & $\begin{array}{l}\text { Chishti et al. }{ }^{15} \\
\text { Riazuddin et al. }{ }^{17}\end{array}$ \\
\hline & & $1.4(8 / 557)$ & $0.7-2.8$ & \\
\hline MYO6 & DFNB37 & $1.2(3 / 250)$ & $0.4-3.4$ & Ahmed et al. ${ }^{35}$ \\
\hline$R D X$ & DFNB24 & $0.3(2 / 557)$ & $0.1-1.3$ & Khan et al. ${ }^{36}$ \\
\hline
\end{tabular}

Abbreviations: $\mathrm{Cl}$, confidence interval; NCEMB, National Centre of Excellence in Molecular Biology.

a definitive result for $\sim 30 \%$ of Pakistani patients with DFNB4/PDS. Our tetra-primer ARMS assay for p.V239D would be a logical first step in a strategy that includes similar assays for other prevalent alleles, such as p.S90L and p.Q446R. As the majority of NCEMB families are from the Punjab province and speak Punjabi, ${ }^{16}$ the details of a hierarchical SLC26A4 mutation screening strategy may need to be refined for other geographical regions and lingo-ethnic groups within Pakistan.

\section{ELECTRONIC DATABASE INFORMATION}

Primer3; http://frodo.wi.mit.edu/cgi-bin/primer3/primer3_www.cgi/ GenScan server at MIT; http://genes.mit.edu/GENSCAN.html.

\section{ACKNOWLEDGEMENTS}

We are grateful to the NCEMB families whose participation made this study possible. We thank Byung Yoon Choi, Erich Boger and Imran Shabbir for their technical advice and comments on this paper. This study was supported by the Higher Education Commission (HEC), Islamabad, Pakistan; EMRO/WHOCOMSTECH and Ministry of Science and Technology (MoST), Islamabad, Pakistan; the International Center for Genetic Engineering and Biotechnology, Trieste, Italy under project CRP/PAK02-01 (Contract no. 02/013); and NIDCD/ $\mathrm{NIH}$ intramural funds 1 ZO1 DC000039 and 1 ZO1 DC000064.

1 Phelps, P. D., Coffey, R. A., Trembath, R. C., Luxon, L. M., Grossman, A. B., Britton, K. E. et al. Radiological malformations of the ear in Pendred syndrome. Clin. Radiol. 53, 268-273 (1998).

2 Everett, L. A., Glaser, B., Beck, J. C., Idol, J. R., Buchs, A., Heyman, M. et al. Pendred syndrome is caused by mutations in a putative sulphate transporter gene (PDS). Nat. Genet. 17, 411-422 (1997).

3 Friedman, T. B. \& Griffith, A. J. Human nonsyndromic sensorineural deafness. Annu. Rev. Genomics. Hum. Genet. 4, 341-402 (2003).

4 Li, X. C., Everett, L. A., Lalwani, A. K., Desmukh, D., Friedman, T. B., Green, E. D. et al. A mutation in PDS causes non-syndromic recessive deafness. Nat. Genet. 18, 215-217 (1998)

5 Madeo, A. C., Pryor, S. P., Brewer, C., Zalewski, C., King, K., Butman, J. A. et al. Pendred Syndrome. Semin. Hear. 27, 160-170 (2006).

6 Pryor, S. P., Park, H. J., Madeo, A. C. \& Griffith, A. J. Pendred syndrome In: Genetic Hearing Loss (ed Willems P.J.). 75-96 (Marcel Dekker, Inc., New York, 2003).

7 Fraser, G. R. Association of Congenital Deafness with Goitre (Pendred's Syndrome) a Study of 207 Families. Ann. Hum. Genet. 28, 201-249 (1965).

8 Park, H. J., Shaukat, S., Liu, X. Z., Hahn, S. H., Naz, S., Ghosh, M. et al. Origins and frequencies of SLC26A4 (PDS) mutations in east and south Asians: global implications for the epidemiology of deafness. J. Med. Genet. 40, 242-248 (2003). 
9 Kopp, P., Pesce, L., Solis, S. J. Pendred syndrome and iodide transport in the thyroid. Trends. Endocrinol. Metab. 19, 260-268 (2008).

10 Guo, Y. F., Liu, X. W., Guan, J., Han, M. K., Wang, D. Y., Zhao, Y. L. et al. GJB2, SLC26A4 and mitochondrial DNA A1555G mutations in prelingual deafness in Northern Chinese subjects. Acta. Otolaryngol. 128, 297-303 (2008).

11 Tsukamoto, K., Suzuki, H., Harada, D., Namba, A., Abe, S. \& Usami, S. Distribution and frequencies of PDS (SLC26A4) mutations in Pendred syndrome and nonsyndromic hearing loss associated with enlarged vestibular aqueduct: a unique spectrum of mutations in Japanese. Eur. J. Hum. Genet. 11, 916-922 (2003).

12 Dai, P., Li, Q., Huang, D., Yuan, Y., Kang, D., Miller, D. T. et al. SLC26A4 c.919-2A>G varies among Chinese ethnic groups as a cause of hearing loss. Genet. Med. 10, 586-592 (2008).

13 Park, H. J., Lee, S. J., Jin, H. S., Lee, J. O., Go, S. H., Jang, H. S. et al. Genetic basis of hearing loss associated with enlarged vestibular aqueducts in Koreans. Clin. Genet. 67, 160-165 (2005).

14 Ahmed, Z. M., Riazuddin, S., Khan, S., Friedman, P., Riazuddin, S., Friedman, T. USH1H, a novel locus for type I Usher syndrome, maps to chromosome 15q22-23. Clin. Genet. 75, 86-91 DOI: 10.1111/j.1399-0004.2008.01038.x (2008).

15 Chishti, M. S., Bhatti, A., Tamim, S., Lee, K., McDonald, M. L., Leal, S. M. et al. Splice-site mutations in the TRIC gene underlie autosomal recessive nonsyndromic hearing impairment in Pakistani families. J. Hum. Genet. 53, 101-105 (2008).

16 Kitajiri, S. I., McNamara, R., Makishima, T., Husnain, T., Zafar, A. U., Kittles, R. A. et al. Identities, frequencies and origins of TMC1 mutations causing DFNB7/B11 deafness in Pakistan. Clin. Genet. 72, 546-550 (2007).

17 Riazuddin, S., Ahmed, Z. M., Fanning, A. S., Lagziel, A., Kitajiri, S., Ramzan, K. et al. Tricellulin is a tight-junction protein necessary for hearing. Am. J. Hum. Genet. 79, 1040-1051 (2006a).

18 Riazuddin, S., Khan, S. N., Ahmed, Z. M., Ghosh, M., Caution, K., Nazli, S. et al. Mutations in TRIOBP, which encodes a putative cytoskeletal-organizing protein, are associated with nonsyndromic recessive deafness. Am. J. Hum. Genet. 78, 137-143 (2006b).

19 Shabbir, M. I., Ahmed, Z. M., Khan, S. Y., Riazuddin, S., Waryah, A. M., Khan, S. N. et al. Mutations of human TMHS cause recessively inherited non-syndromic hearing loss. J. Med. Genet. 43, 634-640 (2006).

20 Ahmed, Z. M., Riazuddin, S., Bernstein, S. L., Ahmed, Z., Khan, S., Griffith, A. J. et al. Mutations of the protocadherin gene PCDH15 cause Usher syndrome type 1F. Am. J. Hum. Genet. 69, 25-34 (2001).

21 Krawczak, M., Reiss, J. \& Cooper, D. N. The mutational spectrum of single base-pair substitutions in mRNA splice junctions of human genes: causes and consequences. Hum. Genet. 90, 41-54 (1992).

22 Audrezet, M. P., Chen, J. M., Raguenes, O., Chuzhanova, N., Giteau, K., Le Marechal, C. et al. Genomic rearrangements in the CFTR gene: extensive allelic heterogeneity and diverse mutational mechanisms. Hum. Mutat. 23, 343-357 (2004).
23 Chen, J. M., Chuzhanova, N., Stenson, P. D., Ferec, C. \& Cooper, D. N. Meta-analysis of gross insertions causing human genetic disease: novel mutational mechanisms and the role of replication slippage. Hum. Mutat. 25, 207-221 (2005).

24 Magnani, C., Cremonesi, L., Giunta, A., Magnaghi, P., Taramelli, R., Ferrari, M. Short direct repeats at the breakpoints of a novel large deletion in the CFTR gene suggest a likely slipped mispairing mechanism. Hum. Genet. 98, 102-108 (1996).

25 Niel, F., Martin, J., Dastot-Le, M. F., Costes, B., Boissier, B., Delattre, V. et al. Rapid detection of CFTR gene rearrangements impacts on genetic counselling in cystic fibrosis. J. Med. Genet. 41, e118 (2004).

26 Reardon, W., CF, O. M., Trembath, R., Jan, H. \& Phelps, P. D. Enlarged vestibular aqueduct: a radiological marker of Pendred syndrome, and mutation of the PDS gene. Q. J. Med. 93, 99-104 (2000).

27 Dai, P., Han, D. Y. \& Wu, B. L. Molecular diagnostic strategies of EVAS in China and US. Otolaryng. Head. Neck. 137, 138 (2007).

28 Usami, S., Wagatsuma, M., Fukuoka, H., Suzuki, H., Tsukada, K., Nishio, S. et al. The responsible genes in Japanese deafness patients and clinical application using Invader assay. Acta. Otolaryngol. 128, 446-454 (2008).

29 Santos, R. L., Wajid, M., Pham, T. L., Hussan, J., Ali, G., Ahmad, W. et al. Low prevalence of Connexin 26 (GJB2) variants in Pakistani families with autosomal recessive non-syndromic hearing impairment. Clin. Genet. 67, 61-68 (2005).

30 Borck, G., Roth, C., Martine, U., Wildhardt, G. \& Pohlenz, J. Mutations in the PDS gene in German families with Pendred's syndrome: V138F is a founder mutation. J. Clin. Endocrinol. Metab. 88, 2916-2921 (2003).

31 Wang, Q. J., Zhao, Y. L., Rao, S. Q., Guo, Y. F., Yuan, H., Zong, L. et al. A distinct spectrum of SLC26A4 mutations in patients with enlarged vestibular aqueduct in China. Clin. Genet. 72, 245-254 (2007).

32 Wu, C. C., Yeh, T. H., Chen, P. J. \& Hsu, C. J. Prevalent SLC26A4 mutations in patients with enlarged vestibular aqueduct and/or Mondini dysplasia: a unique spectrum of mutations in Taiwan, including a frequent founder mutation. Laryngoscope. 115, 1060-1064 (2005).

$33 \mathrm{Nal}$, N., Ahmed, Z. M., Erkal, E., Alper, O. M., Luleci, G., Dinc, O. et al. Mutational spectrum of MYO15A: the large N-terminal extension of myosin XVA is required for hearing. Hum. Mutat. 28, 1014-1019 (2007).

34 Choi, B. Y., Ahmed, Z. M., Riazuddin, S., Bhinder, M. A., Shahzad, M., Husnain, T. et al. Identities and frequencies of mutations of the otoferlin gene (OTOF) causing DFNB9 deafness in Pakistan. Clin. Genet. 75, 237-243 (2009).

35 Ahmed, Z. M., Morell, R. J., Riazuddin, S., Gropman, A., Shaukat, S., Ahmad, M. M. et al. Mutations of MYO6 are associated with recessive deafness, DFNB37. Am. J. Hum. Genet. 72, 1315-1322 (2003).

36 Khan, S. Y., Ahmed, Z. M., Shabbir, M. I., Kitajiri, S., Kalsoom, S., Tasneem, S. et al. Mutations of the RDX gene cause nonsyndromic hearing loss at the DFNB24 locus. Hum. Mutat. 28, 417-423 (2007).

Supplementary Information accompanies the paper on Journal of Human Genetics website (http://www.nature.com/jhg) 